\title{
BMJ Open Randomised trial of mitral valve repair with leaflet resection versus leaflet preservation on functional mitral stenosis (The CAMRA CardioLink-2 Trial)
}

\author{
Vincent Chan, ${ }^{1,2}$ Michael W A Chu, ${ }^{3}$ Howard Leong-Poi, ${ }^{4,5,6,7}$ David A Latter, ${ }^{8,9}$ \\ Judith Hall, ${ }^{10}$ Kevin E Thorpe, ${ }^{10,11}$ Benoit E de Varennes, ${ }^{12}$ Adrian Quan, ${ }^{5,6,8}$ \\ Wendy Tsang, ${ }^{7,13}$ Natasha Dhingra, ${ }^{8}$ Kibar Yared, ${ }^{14}$ Hwee Teoh, $, 6,8,15$ F Victor Chu, ${ }^{16}$ \\ Kwan-Leung Chan, ${ }^{17}$ Thierry G Mesana, ${ }^{1}$ Kim A Connelly, ${ }^{4,5,6,7}$ Marc Ruel, ${ }^{1,2}$ \\ Peter Jüni, ${ }^{10,18}$ C David Mazer, ${ }^{6,7,19}$ Subodh Verma ${ }^{5,6,8,9}$
}

To cite: Chan V, Chu MWA, Leong-Poi $\mathrm{H}$, et al. Randomised trial of mitral valve repair with leaflet resection versus leaflet preservation on functional mitral stenosis (The CAMRA CardioLink-2 Trial). BMJ Open 2017;0:e015032. doi:10.1136/ bmjopen-2016-015032

- Prepublication history and additional material are available. To view these files please visit the journal online (http://dx.doi. org/10.1136/bmjopen-2016015032).

Received 4 November 2016 Revised 16 February 2017 Accepted 23 March 2017

\section{CrossMark}

For numbered affiliations see end of article.

Correspondence to

Dr Subodh Verma;

vermasu@smh.ca

\section{ABSTRACT}

Background The gold-standard treatment of severe mitral regurgitation (MR) due to degenerative disease is valve repair, which is surgically performed with either a leaflet resection or leaflet preservation approach. Recent data suggest that functional mitral stenosis (MS) may occur following valve repair using a leaflet resection strategy, which adversely affects patient prognosis. A randomised comparison of these two approaches to mitral repair on functional MS has not been conducted.

Methods and analysis This is a prospective, multicentre randomised controlled trial designed to test the hypothesis that leaflet preservation leads to better preservation of mitral valve geometry, and therefore, will be superior to leaflet resection for the primary outcome of functional MS as assessed by 12-month mean mitral valve gradient at peak exercise. Eighty-eight patients with posterior leaflet prolapse will be randomised intraoperatively once deemed by the operating surgeon to feasibly undergo mitral repair using either a leaflet resection or leaflet preservation approach. Secondary end points include comparison of repair strategies with regard to mitral valve orifice area, leaflet coaptation height, 6 min walk test and a composite major adverse event end point consisting of recurrent MR $\geq 2+$, death or hospital readmission for congestive heart failure within 12 months of surgery.

Ethics and dissemination Institutional ethics approval has been obtained from all enrolling sites. Overall, there remains clinical equipoise regarding the mitral valve repair strategy that is associated with the least likelihood of functional MS. This trial hopes to introduce high-quality evidence to help surgical decision making in this context. Trial registration number NCT02552771.

\section{INTRODUCTION}

Mitral valve prolapse affects $\sim 2 \%$ of individuals, and many will go on to develop severe mitral regurgitation (MR). ${ }^{1-3}$ These patients are generally young, healthy and with few comorbid conditions. ${ }^{1-6}$ The gold-standard therapy is mitral valve repair, as opposed to

\section{Strengths and limitations}

- Novel randomised trial comparing the two techniques used to repair degenerative mitral regurgitation.

Multicentre study.

- Detailed intermediate-term postoperative echocardiographic assessment following mitral valve repair.

- Relatively small sample size.

- Study includes only patients with posterior leaflet prolapse.

- Study end points will be assessed 12 months following surgery; therefore, the long-term impact of resection or non-resection-based mitral repair will not be evaluated.

replacement, which restores life expectancy and improves symptoms. ${ }^{4-13}$ The mitral valve is generally repaired with two techniques involving either leaflet resection or leaflet preservation using artificial neochordae (figure 1). ${ }^{8-13}$ Importantly, the decision to employ either surgical strategy is largely based on surgeon preference, ${ }^{14}$ and data describing outcomes following mitral repair using either strategy have focused primarily on the development of recurrent MR. ${ }^{6-13}$

Several expert centres have reported excellent mitral valve repair rates and survival with either strategy, ${ }^{4-13}$ but few data are available directly comparing leaflet resection with preservation techniques. In general, most studies are long-term follow-up single centre or surgeon experience of long-term freedom from MR. ${ }^{15-17}$ More importantly, there have been no randomised trials comparing surgical repair approaches with respect to functional mitral stenosis (MS). 

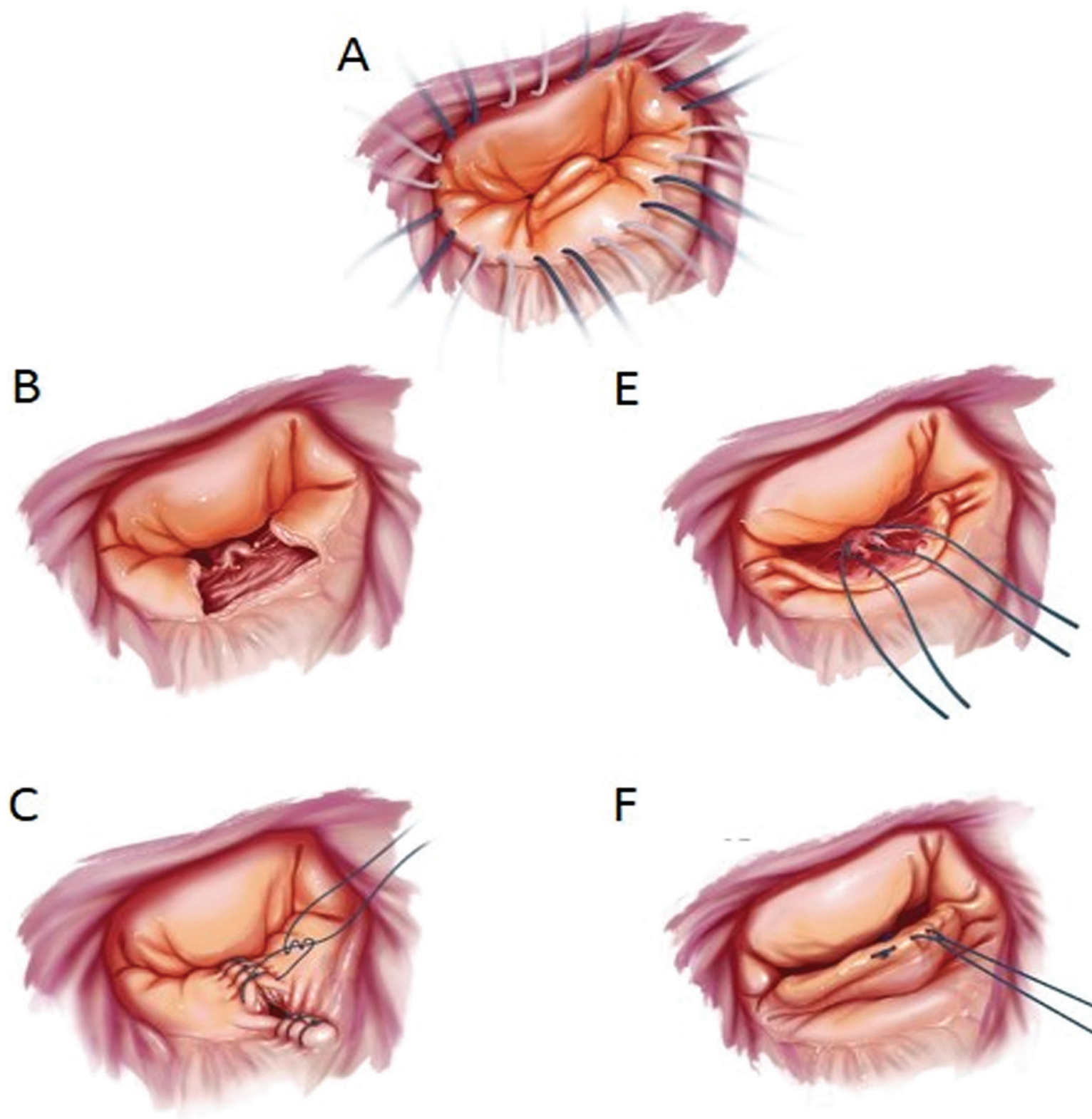

$\mathrm{F}$
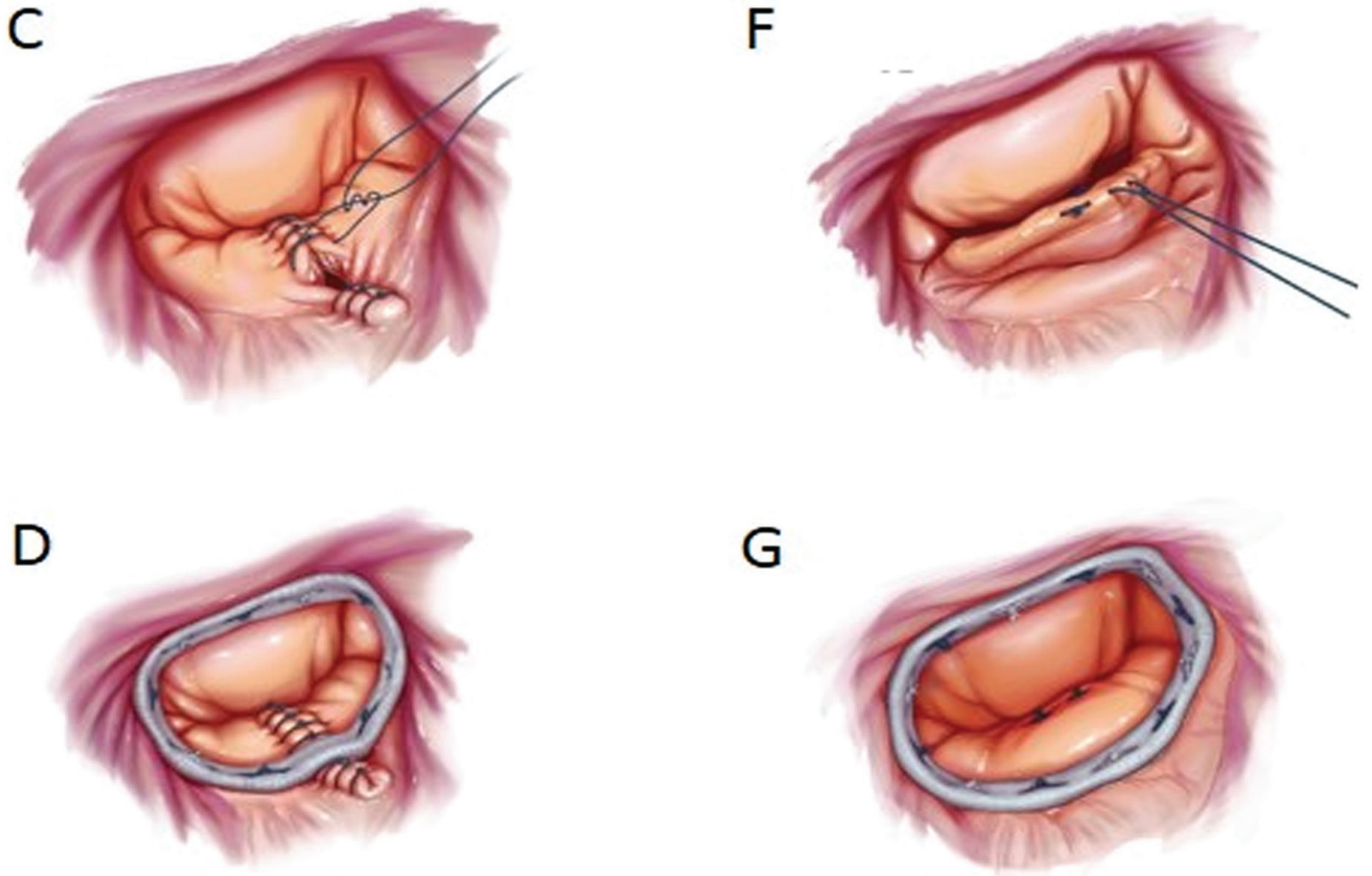

Figure 1 Mitral valve repair using leaflet resection and leaflet preservation techniques. (A) Prolapse of the posterior leaflet of the mitral valve. (B-D) Quadrangular resection of the prolapsing scallop, annular plication and subsequent reconstruction of the remaining lateral and medial edges of the posterior leaflet. $(E-G)$ Valve repair with leaflet preservation via placement of artificial neochordae from the papillary muscles to the prolapsing leaflet edge. 
Emerging data demonstrate that the presence of MS with physiological stress after repair is associated with functional limitations and heart failure even in the absence of recurrent MR. ${ }^{18}$ In a recent study, patients who predominantly had a leaflet resection strategy had a higher peak and mitral valve gradient at peak exercise than patients who predominantly had a leaflet preservation strategy. ${ }^{18}$ Notably, pulmonary artery systolic pressure was also lower in the latter group. ${ }^{18}$ Differences in functional performance were observed also between groups. Patients who predominantly received a leaflet preservation strategy were able to generate more power at peak exercise and achieved a higher metabolic equivalent (MET) score. Beyond this, serum B-type natriuretic peptide (BNP) levels and Short Form (SF)-36 testing were better in patients who had a leaflet preservation strategy at the time of mitral valve repair. These data, though prospective, were derived from a relatively small sample size, and subject to considerable selection bias, argue that mitral valve leaflet preservation may be associated with reduced functional MS and better long-term outcomes.

The presence of functional MS following repair is important since mitral repair is now recommended in selected patients with minimal or no symptoms. ${ }^{19}{ }^{20}$ In spite of the widespread need and performance of mitral repair, randomised trials in this area are lacking, and surgical decision making is often driven by expertise, experience, anecdotes and dogma. We are therefore conducting a novel, prospective randomised study comparing mitral repair of degenerative MR using either a leaflet resection or leaflet preservation approach. We hypothesise that a strategy of mitral valve leaflet preservation leads to better preservation of mitral valve geometry, and therefore, will be superior to leaflet resection for the primary outcome of functional MS as assessed by 12-month mean mitral valve gradient at peak exercise.

\section{METHODS AND ANALYSIS}

\section{Study design summary}

This is a multicentre, non-blinded, double-armed, randomised controlled trial comparing two different surgical strategies for repair of mitral valve prolapse. Patients will be randomly allocated 1:1 to undergo either a leaflet resection or a leaflet preservation strategy (figure 2). Patient screening and consent will be performed by study coordinators at each of the enrolling sites.

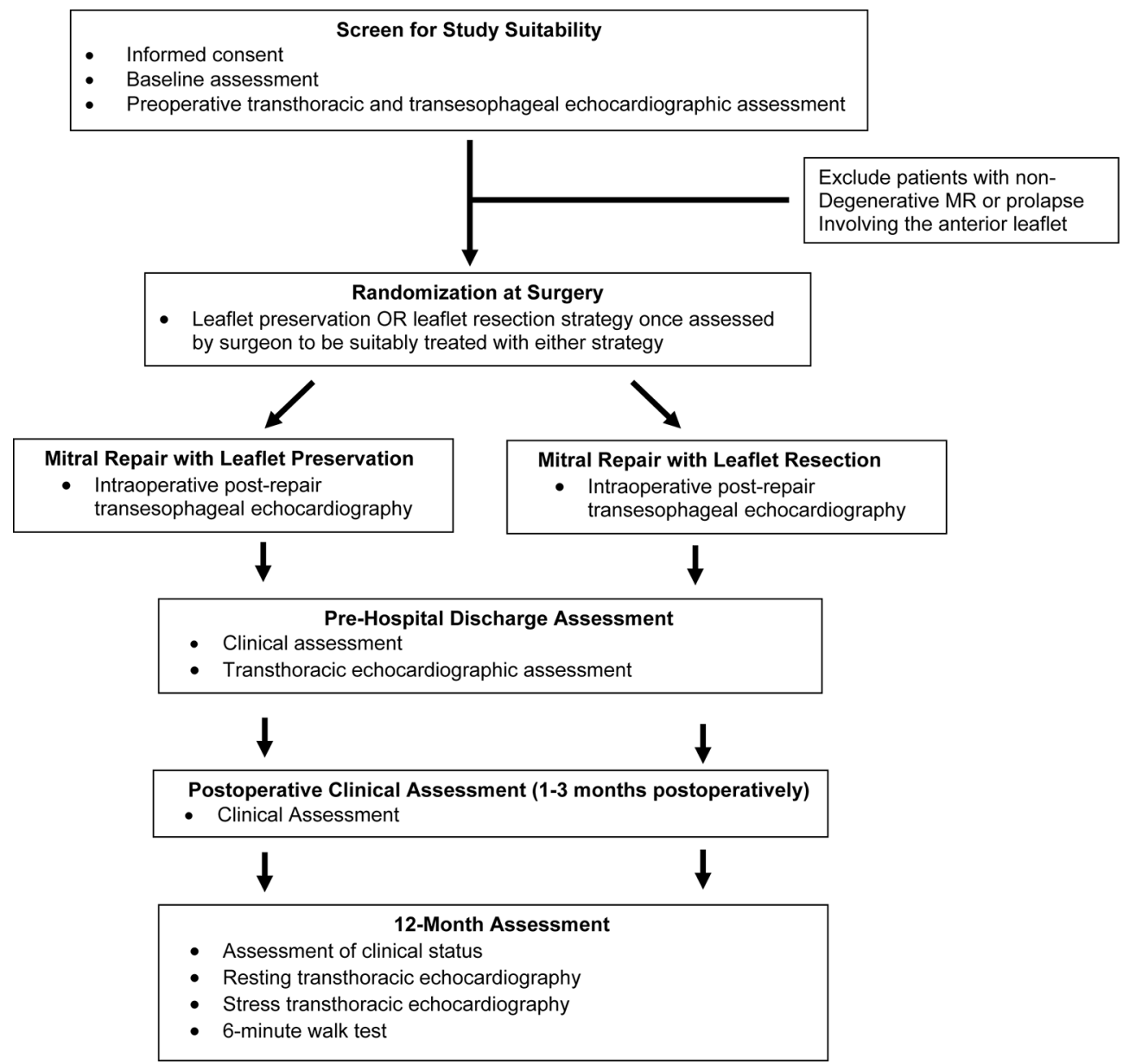

Figure 2 Study schematic. Patients will be assessed clinically and echocardiographically prior to hospital discharge and 1 year following mitral valve reconstruction. 


\section{Study end points}

The purpose of this study is to compare outcomes following repair of degenerative MR using either a leaflet resection or leaflet preservation strategy. The primary objective for this study is to compare mitral repair strategies with regard to mean mitral valve gradient at peak exercise 12 months after surgical repair of mitral valve prolapse.

The secondary objective of this study is to compare leaflet preservation and resection strategies 12 months following surgery with regard to mitral valve orifice area, age-gender predicted MET, mitral leaflet coaptation height, 6 min walk test and a composite major adverse event end point consisting of recurrent $\mathrm{MR} \geq 2+$, death or hospital readmission for congestive heart failure within 12 months of surgery.

\section{Study management}

This trial is funded by the Heart \& Stroke Foundation (Project G-16-00014666) and the CardioLink Trial Platform at St Michael's Hospital, Toronto, Ontario, Canada. The trial is registered at clinicaltrials.gov Identifier: NCT02552771. These funds are unrestricted, therefore, ultimate authority with regard to publication resides with the study authors.

All study data will be de-identified and sent to the Li Ka Shing Knowledge Institute where this information will be secured stored on electronic servers. This includes echocardiographic data, which is read by the independent core echocardiographic laboratory. Access to the final study data set will be restricted to the study principal investigators (VC, SV) and the statisticians involved.

\section{Study population}

Patients will be included in this study if they have posterior mitral valve prolapse amenable to either a leaflet resection or leaflet preservation surgical repair strategy.

Patients will be excluded if they have anterior leaflet or commissural prolapse, endocarditis or rheumatic mitral valve disease, mitral annular calcification extending beyond the circumference of one leaflet scallop, significant left ventricular (LV) dysfunction defined as a LV ejection fraction $<40 \%$, requiring concomitant aortic valve surgery or if they are unable to undergo bicycle ergometry.

Patients with concomitant atrial fibrillation or those who undergo a concomitant Maze procedure or bypass grafting will be included in this study.

\section{Randomisation}

Randomisation will occur intraoperatively following the initial assessment of the mitral valve with the heart arrested while supported on full cardiopulmonary bypass. Randomisation will not occur earlier as the surgeon must be sure that successful valve repair can be safely performed with either a leaflet resection or a leaflet preservation strategy.
After eligibility has been confirmed and the baseline visit assessments completed, patients will be randomly allocated in a 1:1 ratio to receive either a mitral leaflet resection or leaflet preservation surgery. Randomisation will be centralised and generated by the Applied Health Research Centre at the Li Ka Shing Knowledge Institute of St Michael's Hospital. Randomisation will be stratified by centre using random permuted blocks of varying sizes.

\section{Surgical strategy}

The surgical strategy will be standardised among the enrolling mitral surgeons/centres. All mitral valve repairs will be performed either via sternotomy or right thoracotomy with cardioplegic arrest and cardiopulmonary bypass. Only complete annuloplasty with the Carpentier-Edwards Physio II Ring (Edwards Lifesciences, Irivine, California, USA) bands will be used and sizing will be based on the size of the anterior mitral leaflet. Closure of clefts and transfer of in situ chordae may be permitted per surgeon preference. However use of an edge-to-edge repair, either placed centrally or towards either commissure or folding plasty will be considered protocol deviations. The leaflet resection strategy may include either a triangular or quadrangular resection with or without concomitant sliding plasty. The leaflet preservation strategy will include use of either $4-0$ or $5-0$ polytetrafluoroethylene sutures placed on the head of the anterolateral or posteromedial papillary muscle. Use of pledgets for placement of these neochordae on the papillary muscle will be permitted.

\section{Echocardiographic assessment}

All resting echocardiographic measurements will be performed in accordance with current guidelines. ${ }^{21} 22$ In brief, the degree of MR following mitral repair will be assessed through calculation of the effective regurgitation orifice area as determined via the proximal isovelocity surface area method. Estimation of the diastolic pressure gradient across the mitral valve following repair will be assessed by the transmitral velocity flow curve using the simplified Bernouilli equation. Continuous wave Doppler will be used to ensure maximal velocities are recorded and Doppler gradients will be measured in the apical window. Mitral valve area will be measured using planimetry obtained on a parasternal short-axis view in mid-diastole and also via the continuity method. ${ }^{22}$ Additional echocardiographic measurements will be performed to assess changes in left and right ventricular size and systolic function, as per current guidelines. ${ }^{21}$

For stress echocardiographic assessments, patients will be securely positioned on a supine cycle ergometer table that allows for $\mathrm{a} \leq 40^{\circ}$ tilt. Patients will pedal against a fixed resistance. After an initial workload of $25 \mathrm{~W}$ maintained for $2 \mathrm{~min}$, the workload will be increased stepwise by 25 $\mathrm{W}$ every $2 \mathrm{~min}$. Patients will be encouraged to exercise to exhaustion. $^{18}$

All postoperative echocardiographic assessments will be read in a blinded fashion by an independent Core laboratory based at St Michael's Hospital, Toronto. 
Table 1 Study sample size estimates

\section{$10 \%$}

\begin{tabular}{|c|c|c|c|}
\hline & $0 \%$ attrition & $5 \%$ attrition & attrition \\
\hline \multicolumn{4}{|l|}{ Power=90\% } \\
\hline Two tailed & $\begin{array}{l}78 \text { (39 per } \\
\text { group) }\end{array}$ & $\begin{array}{l}84 \text { ( } 42 \text { per } \\
\text { group) }\end{array}$ & $\begin{array}{l}88 \text { (44 per } \\
\text { group) }\end{array}$ \\
\hline \multicolumn{4}{|l|}{ Power $=80 \%$} \\
\hline Two tailed & $\begin{array}{l}60 \text { (30 per } \\
\text { group) }\end{array}$ & $\begin{array}{l}64 \text { (32 per } \\
\text { group) }\end{array}$ & $\begin{array}{l}68 \text { (34 per } \\
\text { group) }\end{array}$ \\
\hline
\end{tabular}

\section{Study sample size}

Stress echocardiography data following repair of degenerative MR has not been commonly reported. However, we have previously determined mean mitral gradients at peak exercise in selected patients who underwent mitral repair using a combination of leaflet preservation and resection techniques. ${ }^{1823}$ Based on these data and considering current valve guidelines, we propose a $5 \mathrm{~mm} \mathrm{Hg}$ difference in mean mitral valve gradient at peak exercise to be clinically significant. Considering an SD of $6.7 \mathrm{~mm}$ $\mathrm{Hg}$ based on our previous data, ${ }^{18} 88$ patients would be required to detect a difference between groups using a two-sided test with $5 \%$ alpha, $90 \%$ power and $10 \%$ patient attrition (table 1).

This study will be conducted at four tertiary-care cardiac surgery centres with a combined annual case volume of approximately 5500 operations. Of these, approximately 300 operations are for degenerative MR due to posterior leaflet prolapse, therefore, study enrolment appears feasible to be completed within 2 years of site initiation.

\section{Data analysis}

Baseline characteristics will be compared between groups using a $\chi^{2}$ test for categorical variables or a Student's t-test for continuous variables. The primary outcome and continuous secondary outcomes will be compared between groups using a Student's t-test. The treatment effect will be expressed as the mean difference between groups with $95 \%$ CI. Missing data for the primary outcome is unlikely to be missing at random and so standard imputation approaches are problematic. Therefore, two analyses will be conducted if the primary outcome is missing in more than $5 \%$ of the randomised subjects. The first will be the usual complete case analysis. The second will employ inverse probability weighting on the probability of 'completing' the study. If these analyses are concordant, the simpler analysis will be primary. The proportion of individuals experiencing the composite major adverse cardiac end point of recurrent $\mathrm{MR} \geq 2+$, death or hospital readmission for congestive heart failure within 12 months of surgery will be compared between groups using method $\chi^{2}$ test. Risk factors associated with the composite end point will also be assessed by logistic regression to determine the adjusted impact of the mitral repair strategy on outcomes. A two-sided significance level of $5 \%$ will be used throughout. Statistical analysis will be performed using $\mathrm{R}^{24}$ The results from the echocardiographic assessments performed 12 months following surgery will be reported to the site investigators so as to inform patient care decisions. This study is powered to tolerate a $10 \%$ patient attrition or protocol non-adherence. Therefore, if attrition exceeds $10 \%$, more patients will be recruited to ensure adequate study power.

In addition to the reporting of study end points, the overall number of patients undergoing repair of degenerative MR at each treatment centre will be reported to better provide context of the findings of the study.

\section{Study retention and safety}

Study patients will be informed of the multiple postoperative assessments prior to enrolment to maximise study retention. Study coordinators will also work with the individual surgeon offices to ensure patient follow-up. A separate data safety and monitoring committee will evaluate surgeon and surgical site repair rates to ensure that there is no negative impact of repair intervention on clinical outcomes. This will involve review of intraoperative postrepair echocardiograms in addition to echocardiograms performed prior to hospital discharge following surgery. Nevertheless, the intervention is considered low risk given the surgical expertise an the fact that patients will be managed according to current guidelines and practice standards. ${ }^{19} 20$ Notwithstanding, information from the additional postoperative echocardiographic assessments will be returned to each treating surgeon's office to updated clinical status data.

\section{ETHICS AND DISSEMINATION}

Mitral valve repair, as opposed to replacement, is the gold-standard treatment of severe MR due to leaflet prolapse. $^{1-5}$ Although leaflet resection and leaflet preservation techniques have been well described, no randomised data are available comparing these two approaches with regard to functional MS. These data may guide clinical practice, which currently involves use of a given technique based on surgeon preference. If the hypothesis that a leaflet preservation technique results in less functional stenosis, this will lead to less leaflet resection techniques employed in mitral valve reconstruction. This may have particular relevance for young patients who undergo mitral reconstruction who are able to attain higher output states at exercise. ${ }^{13} 19$

In previous work performed by Chan $\mathrm{K}$ et $a l,{ }^{18} 110$ patients who underwent repair of MR due to myxomatous degeneration were divided into those that had a mean intraoperative mitral gradient $\leq 3 \mathrm{~mm} \mathrm{Hg}$ and $>3 \mathrm{~mm} \mathrm{Hg}$. Patients with a higher mean transmitral repair gradient were more likely to undergo leaflet resection with annular plication. These patients were subjected to stress echocardiography via bicycle ergometry, and serum BNP analysis, 6 min walk test and SF-36 assessments at a mean of 4.2 2.3 years after surgery were performed. Patients that received a predominantly leaflet resection strategy had a higher 
peak (24.8 \pm 10.2 vs $15.6 \pm 6.4 \mathrm{~mm} \mathrm{Hg}, \mathrm{p}<0.001)$ and mean $(14.2 \pm 7.1 \mathrm{vs} 8.9 \pm 3.8 \mathrm{~mm} \mathrm{Hg}, \mathrm{p}<0.001)$ mitral valve gradient at peak exercise compared with patients who underwent a predominantly leaflet preservation repair strategy. Notably, differences in functional performance were observed between groups. Patients who predominantly received a leaflet preservation strategy were able to generate more power at peak exercise and achieved a higher MET score. Beyond this, serum BNP levels and SF-36 testing was better in patients who had a leaflet preservation strategy at the time of mitral valve repair. ${ }^{18}$ However, our work included patients who underwent mitral repair with a blend of leaflet resection and preservation strategies. Also, patients underwent stress echocardiography and functional assessment years after surgery. Thus, although these data suggest that a mitral leaflet preservation strategy may result in less functional MS than repair with leaflet resection, this hypothesis needs validation.

This proposed randomised study represents the first trial comparing mitral repair techniques considering functional MS. Notwithstanding, there are important limitations of this trial. Patients will not be enrolled if they have complex lesions involving the anterior leaflet or MR due to non-degenerative causes. Furthermore, our primary outcome is based on echocardiographic assessments 1 year after surgery; therefore, the longterm durability of different repair techniques will not be assessed. Conclusions regarding the performance of resection or non-resection techniques in surgeons less familiar with mitral repair will also remain unknown.

This study involves surgeons and centres familiar with mitral valve reconstruction, thereby minimising the risk to the patient. ${ }^{6}$ Also, randomisation will be performed only after the operating surgeon has deemed that successful valve repair can be performed using either leaflet resection or leaflet preservation techniques. In this study, patients will be subjected to several postoperative echocardiographic assessments, which goes beyond the structure of follow-up typically performed at most operating centres.

Overall, this proposed prospective trial will provide randomised data comparing the two widely used techniques for repair of degenerative MR. It is our hope that data from this trial will help guide clinical practice and the care of the numerous patients who undergo mitral reconstruction annually through the ultimate publication and presentation of the study results.

\section{CONCLUSION}

The findings from this study will further refine clinical mitral repair practice. As yet, there remains no randomised data to comprehensively advise surgeons as to which strategy to repair mitral prolapse, whether leaflet resection or preservation. Data from this study highlights the importance of mitral valve repair in these young patients who may experience functional limitations with an imperfect mitral reconstruction. Furthermore, it is the goal of the researchers to underscore the importance of mitral valve reconstruction in these patients.

\section{Author affiliations}

${ }^{1}$ Division of Cardiac Surgery, University of Ottawa Heart Institute, Ottawa, Ontario, Canada

${ }^{2}$ School of Epidemiology, Public Health and Preventive Medicine, University of Ottawa, Ottawa, Ontario, Canada

${ }^{3}$ Division of Cardiac Surgery, London Health Sciences Center, University of Western Ontario, London, Ontario, Canada

${ }^{4}$ Division of Cardiology, St Michael's Hospital, Toronto, Ontario, Canada

${ }^{5}$ Keenan Research Centre for Biomedical Science, St Michael's Hospital, Toronto, Ontario, Canada

${ }^{6}$ Li Ka Shing Knowledge Institute, St Michael's Hospital, Toronto, Ontario, Canada

${ }^{7}$ Department of Medicine, University of Toronto, Toronto, Ontario, Canada

${ }^{8}$ Division of Cardiac Surgery, St Michael's Hospital, University of Toronto, Toronto, Ontario, Canada

${ }^{9}$ Department of Surgery, University of Toronto, Toronto, Ontario, Canada

${ }^{10}$ Applied Health Research Centre, St Michael's Hospital, Toronto, Ontario, Canada

${ }^{11}$ Dalla Lana School of Public Health, University of Toronto, Toronto, Ontario, Canada

${ }^{12}$ Division of Cardiac Surgery, Royal Victoria Hospital, McGill University Health Center, Montreal, Québec, Canada

${ }^{13}$ Division of Cardiology, University Health Network, Toronto, Ontario, Canada

${ }^{14}$ Division of Cardiology, The Scarborough Hospital, Toronto, Ontario, Canada

${ }^{15}$ Division of Endocrinology and Metabolism, St Michael's Hospital, Toronto, Ontario, Canada

${ }^{16}$ Department of Cardiac Surgery, McMaster University, Hamilton, Ontario, Canada

${ }^{17}$ Division of Cardiology, University of Ottawa Heart Institute, Ottawa, Ontario, Canada

${ }^{18}$ Institute of Health Policy, Management and Evaluation, University of Toronto, Toronto, Ontario, Canada

${ }^{19}$ Department of Anesthesia, St Michael's Hospital, Toronto, Ontario, Canada

Contributors VC and SV drafted the manuscript. All authors reviewed and critically revised the manuscript and approved the final version of the submitted manuscript.

Competing interests MWAC: consultant to Edwards Lifesciences, Medtronic Canada, Livanova and Symetis. PJ: received research grants to the institution from AstraZeneca, Biotronik, Biosensors International, Eli Lilly and The Medicines Company, and serves as unpaid member of the steering group of trials funded by AstraZeneca, Biotronik, Biosensors, St Jude Medical and The Medicines Company. All other authors report no conflicts of interest.

Ethics approval St Michael's Hospital.

Provenance and peer review Not commissioned; externally peer reviewed.

Open Access This is an Open Access article distributed in accordance with the Creative Commons Attribution Non Commercial (CC BY-NC 4.0) license, which permits others to distribute, remix, adapt, build upon this work non-commercially, and license their derivative works on different terms, provided the original work is properly cited and the use is non-commercial. See: http://creativecommons.org/ licenses/by-nc/4.0/

(c) Article author(s) (or their employer(s) unless otherwise stated in the text of the article) 2017. All rights reserved. No commercial use is permitted unless otherwise expressly granted.

\section{REFERENCES}

1. Theal M, Sleik K, Anand S, et al. Prevalence of mitral valve prolapse in ethnic groups. Can J Cardiol 2004;20:511-5.

2. Freed LA, Levy D, Levine RA, et al. Prevalence and clinical outcome of mitral-valve prolapse. N Engl J Med 1999;341:1-7.

3. Flack JM, Kvasnicka JH, Gardin JM, et al. Anthropometric and physiologic correlates of mitral valve prolapse in a biethnic cohort of young adults: the CARDIA study. Am Heart J 1999;138:486-92.

4. Chan V, Ruel M, Elmistekawy E, et al. Determinants of left ventricular dysfunction after repair of chronic asymptomatic mitral regurgitation. Ann Thorac Surg 2015;99:38-42.

5. Chan V, Ruel M, Hynes M, et al. Impact of mitral annular calcification on early and late outcomes following mitral valve repair of myxomatous degeneration. Interact Cardiovasc Thorac Surg 2013;17:120-5. 
6. Chan V, Ruel M, Chaudry S, et al. Clinical and echocardiographic outcomes after repair of mitral valve bileaflet prolapse due to myxomatous disease. J Thorac Cardiovasc Surg 2012;143:S8-S11.

7. Castillo JG, Anyanwu AC, Fuster V, et al. A near $100 \%$ repair rate for mitral valve prolapse is achievable in a reference center: implications for future guidelines. J Thorac Cardiovasc Surg 2012;144:308-12.

8. Carpentier A. Cardiac valve surgery-the "French correction". J Thorac Cardiovasc Surg 1983;86:323-37.

9. Braunberger E, Deloche A, Berrebi A, et al. Very long-term results (more than 20 years) of valve repair with Carpentier's techniques in nonrheumatic mitral valve insufficiency. Circulation 2001;104(12 Suppl 1):18-11.

10. Filsoufi F, Carpentier A. Principles of reconstructive surgery in degenerative mitral valve disease. Semin Thorac Cardiovasc Surg 2007:19:103-10.

11. Ling LH, Enriquez-Sarano M, Seward JB, et al. Clinical outcome of mitral regurgitation due to flail leaflet. N Engl J Med 1996;335:1417-23.

12. Enriquez-Sarano M, Avierinos JF, Messika-Zeitoun D, et al. Quantitative determinants of the outcome of asymptomatic mitral regurgitation. N Engl J Med 2005;352:875-83.

13. Verma S, Mesana TG. Mitral-valve repair for mitral-valve prolapse. $N$ Engl J Med 2009;361:2261-9.

14. Gammie JS, Sheng S, Griffith BP, et al. Trends in mitral valve surgery in the United States: results from the Society of Thoracic Surgeons adult cardiac Surgery database. Ann Thorac Surg 2009;87:1431-9.

15. Seeburger J, Falk V, Borger MA, et al. Chordae replacement versus resection for repair of isolated posterior mitral leaflet prolapse: à ègalité. Ann Thorac Surg 2009;87:1715-20.

16. Falk V, Seeburger J, Czesla M, et al. How does the use of polytetrafluoroethylene neochordae for posterior mitral valve prolapse (loop technique) compare with leaflet resection? A prospective randomized trial. J Thorac Cardiovasc Surg 2008;136:1200-6.
17. Lange R, Guenther T, Noebauer C, et al. Chordal replacement versus quadrangular resection for repair of isolated posterior mitral leaflet prolapse. Ann Thorac Surg 2010;89:1163-70.

18. Chan KL, Chen SY, Chan V, et al. Functional significance of elevated mitral gradients after repair for degenerative mitral regurgitation. Circ Cardiovasc Imaging 2013;6:1041-7.

19. Nishimura RA, Otto CM, Bonow RO, et al. AHA/ACC guideline for the management of patients with valvular heart disease: a report of the American College of Cardiology/American Heart Association Task Force on Practice guidelines. J Thorac Cardiovasc Surg 2014;2014:e1-e132.

20. Vahanian A, Alfieri O, Andreotti F, et al. Guidelines on the management of valvular heart disease (version 2012): the Joint Task Force on the management of valvular Heart disease of the european Society of Cardiology (ESC) and the European Association for Cardio-Thoracic Surgery (EACTS). Eur J Cardiothorac Surg 2012;42:S1-44.

21. Lang RM, Badano LP, Mor-Avi V, et al. Recommendations for cardiac chamber quantification by echocardiography in adults: an update from the American Society of Echocardiography and the European Association of Cardiovascular Imaging. J Am Soc Echocardiogr 2015;28:1-39.

22. Baumgartner $\mathrm{H}$, Hung J, Bermejo J, et al. Echocardiographic assessment of valve stenosis: EAE/ASE recommendations for clinical practice. J Am Soc Echocardiogr 2009;22:1-23.

23. Mesana TG, Lam BK, Chan V, et al. Clinical evaluation of functional mitral stenosis after mitral valve repair for degenerative disease: potential affect on surgical strategy. J Thorac Cardiovasc Surg 2013;146:1418-25.

24. R: a language and environment for statistical computing [program]. Vienna, Austria: R Foundation for Statistical Computing, 2016. https://www.R-project.org/. 